\title{
Gauge and Lorentz Covariant Quark Propagator in an Arbitrary Gluon Field
}

\author{
Yong-Hong $\mathrm{An}^{a}$, Hua Yang ${ }^{a, c}$, Qing Wang ${ }^{a, b}$ \\ ${ }^{a}$ Department of Physics, Tsinghua University, Beijing 100084, China * \\ ${ }^{b}$ Institute of Theoretical Physics, Academia Sinica, Beijing 100080, China \\ ${ }^{c}$ Science School, Information Engineering University, Zhengzhou 450004
}

(Feb 7, 2003)

\begin{abstract}
The quark propagator in presence of an arbitrary gluon field is calculated gauge and Lorentz covariantly order by order in terms of powers of gluon field and its derivatives. The result is independent of path connecting ends of propagator and leading order result coincides with the exact propagator in the trivial case of vanishing gluon field.
\end{abstract}

PACS number(s): 11.15.Tk, 12.20.Ds, 12.38.Lg, 14.65.-q

The quark propagator $S(x, y ; A)=(i \not \nabla-m)^{-1}(x, y)^{1}$ with $\nabla^{\mu}=\partial^{\mu}-i g A^{\mu}$ for a quark of mass $m$ in the presence of a gluon field $A^{\mu}$ plays important role in many investigations of quantum chromodynamics. Integrate out gluon field, we get physical quark propagator by

$$
-i\langle 0|\mathbf{T} \psi(x) \bar{\psi}(y)| 0\rangle=\frac{\int \mathcal{D} A_{\mu} S(x, y ; A) e^{i S_{\mathrm{QCD}}(A)}}{\int \mathcal{D} A_{\mu} e^{i S_{\mathrm{QCD}}(A)}} \equiv\langle S(x, y ; A)\rangle,
$$

where $S_{\mathrm{QCD}}(A)$ is QCD effective action with quark field be integrated out. Propagator $S(x, y ; A)$ is Lorentz transformation covariant and under color gauge transformation $A_{\mu}(x) \rightarrow V(x) A_{\mu}(x) V^{\dagger}(x)-i / g V(x)\left[\partial^{\mu} V^{\dagger}(x)\right]$ transforms as

$$
S(x, y ; A) \rightarrow S^{\prime}(x, y ; A)=V(x) S(x, y ; A) V^{\dagger}(y) .
$$

\footnotetext{
${ }^{*}$ Mailing address

${ }^{1}$ Our formulae are given in Minkowski space with Bjorken Drell conventions.
} 
Except the formal expression of $S(x, y ; A)$, an expansion for $S(x, y ; A)$ in terms of local gluon field $A$ is expected and plays a key role in its applications. Note bilocal transformation law (2) prohibit the naive expansion $\sum_{n} C_{n}(x-y) O_{n}[A(x)]$ for $S(x, y ; A)$ with $C_{n}(x-y)$ be gluon field independent coefficient and $O_{n}[A(x)]$ local operator depending on gluon field $A_{\mu}(x)$, since it is impossible for local operator $O_{n}[A(x)]$ transform bilocally. We will set up a modified expansion by multiply naive expansion a local $A_{\mu}(x)$ dependent "face factor" a $[x-y ; A(x)]$ with bilocal transformation law $\mathbf{a}[x-y ; A(x)] \rightarrow \mathbf{a}^{\prime}[x-y ; A(x)]=V(x) \mathbf{a}[x-y ; A(x)] V^{\dagger}(y)$

$$
S(x, y ; A)=\left[\sum_{n} C_{n}(x-y) O_{n}[A(x)]\right] \mathbf{a}[x-y ; A(x)]
$$

which will match the transformation law (2). Then (1) imply we can expand physical quark propagator as,

$$
-i\langle 0|\mathbf{T} \psi(x) \bar{\psi}(y)| 0\rangle=\langle S(x, y ; A)\rangle=\sum_{n} C_{n}(x-y)\left\langle O_{n}[A(x)] \mathbf{a}[x-y, A(x)]\right\rangle,
$$

which can be treated as a gauge covariant modified operator product expansion for quark propagator.

In literature, the most simple approximation for the expansion of $S(x, y ; A)$ is based on the perturbation expansion

$$
\begin{aligned}
S(x, y ; A)= & {\left[\left[1+(i \not \supset-m)^{-1} g \not \mathcal{A}\right]^{-1}(i \not \supset-m)^{-1}\right](x, y) } \\
= & (i \not \supset-m)^{-1}(x, y)-\left[(i \not \supset-m)^{-1} g \not A(i \not \supset-m)^{-1}\right](x, y) \\
& +\left[(i \not \supset-m)^{-1} g \not A(i \not \supset-m)^{-1} g \not A(i \not \supset-m)^{-1}\right](x, y)+\cdots,
\end{aligned}
$$

the expansion can be calculated up to arbitrary orders. The result can be directly expressed in terms of powers of gluon field and its differentials, but to any fixed order of calculation, the gauge covariance is violated. Another approximation is so called static approximation proposed by Brown, Weisberger [1] and Eichten, Feinberg [2]. By neglecting the spatial part of $\not$, it leads

$$
\begin{aligned}
S_{\text {static }}(x, y ; A)= & -\frac{i}{2}\left[\theta\left(x_{0}-y_{0}\right)\left(1+\gamma^{0}\right)+\theta\left(y_{0}-x_{0}\right)\left(1-\gamma^{0}\right)\right] \\
& \times \delta(\vec{x}-\vec{y}) e^{-i m\left|x_{0}-y_{0}\right|} \mathbf{P} e^{i g \int_{y}^{x} d z^{0} A_{0}(z)},
\end{aligned}
$$

where the path in the integral is the straight line from $y$ to $x$, and the path ordering is $A_{0}(x)$ to the left, $\cdots, A_{0}(y)$ to the right. The neglect spatial term can be subsequently taken into account as perturbation [3]. This formalism keeps the gauge covariance of the 
propagator, but Lorentz covariance is lost, it even does not coincide with the exact propagator in the trivial case of vanishing gluon field. Recently, D.Gromes reinvestigate the problem [4]. He, in terms of path ordered exponentials, write the first order perturbation theory as non-perturbative expression which has correct behavior under Lorentz and gauge transformation. His result is only at the lowest order, existence of path ordered exponentials make the expression very complex and cause the problem of path dependence. It is purpose of present work to invent another path independent calculation formalism which can keep the advantages of different formalisms mentioned above,

- The expansion can be calculated up to arbitrary orders.

- The result can be directly expressed in terms of powers of gluon field and its differentials and it coincide with the exact propagator in the trivial case of vanishing gluon field.

- The result is gauge and Lorentz transformation covariant.

- There is no path dependence of the result.

We start by write quark propagator as

$$
S(x, y ; A)=\left\langle x\left|(i \not \nabla+m)\left(E-\nabla^{2}-m^{2}\right)^{-1}\right| y\right\rangle,
$$

where $E-\nabla^{2}-m^{2} \equiv(i \not \nabla-m)(i \not \nabla+m)=(i \not \nabla+m)(i \not \nabla-m)$ with $E=\frac{i g}{4}\left[\gamma^{\mu}, \gamma^{\nu}\right] F_{\mu \nu}$ and $F_{\mu \nu} \equiv \frac{i}{g}\left[\nabla_{\mu}, \nabla_{\nu}\right]=\partial_{\mu} A_{\nu}-\partial_{\nu} A_{\mu}-i g\left[A_{\mu}, A_{\nu}\right]$.

The next step is to calculate matrix element $\left\langle x\left|(i \not \nabla+m)\left(E-\nabla^{2}-m^{2}\right)^{-1}\right| y\right\rangle$ which in momentum space can be written as

$$
\begin{aligned}
& \left\langle x\left|(i \not \nabla+m)\left(E-\nabla^{2}-m^{2}\right)^{-1}\right| y\right\rangle \\
& =\left.\int \frac{d^{4} k}{(2 \pi)^{4}} e^{-i k \cdot z}\left(i \nabla_{x}+\not k+m\right)\left[\left(k+i \nabla_{x}\right)^{2}+E(x)-m^{2}\right]^{-1} 1\right|_{z=x-y} .
\end{aligned}
$$

Conventionally, directly perform Taylor expansion over operator $\nabla_{x}^{\mu}$ and $E(x)$ will leads the result. The gauge covariance in this calculation program is not obvious, since operator $\nabla_{x}^{\mu}$, once acting on the final unity 1 , gives $-i g A^{\mu}(x)$ which is not gauge covariant quantity. Only if $\nabla_{x}^{\mu}$ and $E(x)$ are composed of commutators such as $\left[\nabla_{x}^{\mu}, \nabla_{x}^{\nu}\right]$ and $\left[\nabla_{x}^{\mu}, E(x)\right]$, the gauge covariance is explicit realized. We need a formalism to explicitly exhibit this gauge covariance.

Consider 


$$
e^{i \nabla_{x} \cdot \frac{\partial}{\partial k}} k^{\mu} e^{-i \nabla_{x} \cdot \frac{\partial}{\partial k}}=k^{\mu}+i \nabla_{x}^{\mu}+F\left[\left(i \nabla_{x} \cdot \frac{\partial}{\partial k}\right) d\left(i \nabla_{x} \cdot \frac{\partial}{\partial k}\right)\right]\left(i \nabla_{x}^{\mu}\right)
$$

where we have used relation

$$
\begin{aligned}
e^{A} B e^{-A} & =\left[e^{A d A}\right](B)=B+[A, B]+\frac{1}{2 !}[A,[A, B]]++\frac{1}{3 !}[A,[A,[A, B]]]+\cdots \\
& =B+[A, B]+F[A d A]([A, B])
\end{aligned}
$$

with $F$ function and $(A d A)^{n}$ operation introduced in Ref. [5] are defined as

$$
\begin{aligned}
& F(z) \equiv \frac{e^{z}-1}{z}-1=\sum_{n=2}^{\infty} \frac{z^{n-1}}{n !} \\
& (A d A)^{0}(B) \equiv B \quad(A d A)^{m}(B)=[A,[A, \cdots,[A, B]], \cdots] \quad \text { m times . }
\end{aligned}
$$

(9) can be written as

$$
k^{\mu}+i \nabla_{x}^{\mu}=e^{i \nabla_{x} \cdot \frac{\partial}{\partial k}}\left[k^{\mu}+\tilde{F}^{\mu}\left(\nabla_{x}, \frac{\partial}{\partial k}\right)\right] e^{-i \nabla_{x} \cdot \frac{\partial}{\partial k}},
$$

in which $\tilde{F}^{\mu}\left(\nabla_{x}, \frac{\partial}{\partial k}\right)$ depend on $\nabla_{x}^{\mu}$ and $\frac{\partial}{\partial k}$,

$$
\begin{aligned}
\tilde{F}^{\mu}\left(\nabla_{x}, \frac{\partial}{\partial k}\right) & \equiv-e^{-i \nabla_{x} \cdot \frac{\partial}{\partial k}} F\left[\left(i \nabla_{x} \cdot \frac{\partial}{\partial k}\right) d\left(i \nabla_{x} \cdot \frac{\partial}{\partial k}\right)\right]\left(i \nabla_{x}^{\mu}\right) e^{i \nabla_{x} \cdot \frac{\partial}{\partial k}} \\
& =\frac{1}{2}\left[\nabla_{x}^{\nu}, \nabla_{x}^{\mu}\right] \frac{\partial}{\partial k^{\nu}}-\frac{i}{3}\left[\nabla_{x}^{\lambda},\left[\nabla_{x}^{\nu}, \nabla_{x}^{\mu}\right]\right] \frac{\partial^{2}}{\partial k^{\lambda} \partial k^{\nu}}+O\left(p^{4}\right) .
\end{aligned}
$$

We find all terms in (11) are gauge covariant. For convenient of expansion we assign the each $\nabla_{x}^{\mu}$ a momentum order $p$, then the terms $O\left(p^{4}\right)$ in (11) are those commutators at least with four $\nabla_{x}^{\mu}$ derivatives.

In terms of $\tilde{F}$ function, (10) tells us term $k^{\mu}+i \nabla_{x}^{\mu}$ can be expressed in terms of $\frac{\partial}{\partial k}$ dependent but gauge covariant quantity $\tilde{F}$ multiply some $\frac{\partial}{\partial k}$ dependent exponential "face factors". Apply this to (8),

$$
\begin{aligned}
& \left\langle x\left|(i \not \nabla+m)\left(E-\nabla^{2}-m^{2}\right)^{-1}\right| y\right\rangle \\
& =\left.\int \frac{d^{4} k}{(2 \pi)^{4}} e^{-i k \cdot z} e^{i \nabla_{x} \cdot \frac{\partial}{\partial k}}(\tilde{F} \partial k+\not k+m) e^{-i \nabla_{x} \cdot \frac{\partial}{\partial k}}\left[e^{i \nabla_{x} \cdot \frac{\partial}{\partial k}}\left(k+\tilde{F}_{\partial k}\right)^{2} e^{-i \nabla_{x} \cdot \frac{\partial}{\partial k}}+E(x)-m^{2}\right]^{-1} 1\right|_{z=x-y} \\
& =\left.\int \frac{d^{4} k}{(2 \pi)^{4}} e^{i \nabla_{x} \cdot \frac{\partial}{\partial k}}\left(\tilde{F}_{\partial k}+\not k+m\right)\left[\left(k+\tilde{F}_{\partial k}\right)^{2}+\tilde{E}_{\partial k}(x)-m^{2}\right]^{-1} e^{-i \nabla_{x} \cdot \frac{\partial}{\partial k}} e^{-i k \cdot z}\right|_{z=x-y} \\
& =\left.\int \frac{d^{4} k}{(2 \pi)^{4}}\left(\tilde{F}_{\partial k}+\not k+m\right)\left[\left(k+\tilde{F}_{\partial k}\right)^{2}+\tilde{E}_{\partial k}(x)-m^{2}\right]^{-1} e^{-i k \cdot z} e^{-z \cdot \nabla_{x}}\right|_{z=x-y}
\end{aligned}
$$

where $\nabla_{x}$ commute with $z$. In last equality, we have dropped total momentum space derivative terms 


$$
\int \frac{d^{4} k}{(2 \pi)^{4}}\left[e^{i \nabla_{x} \cdot \frac{\partial}{\partial k}}-1\right]\left[\left(\tilde{F}_{\partial k}+\not k+m\right)\left[\left(k+\tilde{F}_{\partial k}\right)^{2}+\tilde{E}_{\partial k}(x)-m^{2}\right]^{-1} e^{-i k \cdot z} e^{-z \cdot \nabla_{x}}\right]_{z=x-y}
$$

$\tilde{F}_{\partial k}^{\mu} \equiv \tilde{F}^{\mu}\left(\nabla_{x}, \frac{\partial}{\partial k}\right)$ and $\tilde{E}$ are all gauge transformation covariant quantities with $\frac{\partial}{\partial k}$ dependent $\tilde{E}$ be defined as,

$$
\begin{aligned}
\tilde{E}_{\partial k} & \equiv \tilde{E}\left(\nabla_{x}, \frac{\partial}{\partial k}\right)=e^{-i \nabla_{x} \cdot \frac{\partial}{\partial k}} E(x) e^{i \nabla_{x} \cdot \frac{\partial}{\partial k}} \\
& =E(x)-i\left[\nabla_{x}^{\mu}, E(x)\right] \frac{\partial}{\partial k^{\mu}}-\frac{1}{2}\left[\nabla_{x}^{\nu},\left[\nabla_{x}^{\mu}, E(x)\right]\right] \frac{\partial^{2}}{\partial k^{\nu} \partial k^{\mu}}+\cdots
\end{aligned}
$$

(12) imply

$$
S(x, y ; A)=\tilde{S}[x-y ; A(x)] \mathbf{a}[x-y ; A(x)],
$$

with $\tilde{S}[x-y ; A(x)]$ be defined as

$$
\begin{aligned}
\tilde{S}[z ; A(x)] & \equiv \int \frac{d^{4} k}{(2 \pi)^{4}}\left[\tilde{F}_{\partial k}(x)+\not k+m\right]\left[\left(k+\tilde{F}_{\partial k}(x)\right)^{2}+\tilde{E}_{\partial k}(x)-m^{2}\right]^{-1} e^{-i k \cdot z} \\
\mathbf{a}[z ; A(x)] & \equiv e^{-z \cdot \nabla_{x}} 1 .
\end{aligned}
$$

So the quark propagator in presence of gluon field is consists of two parts: one is $\tilde{S}[x-$ $y ; A(x)$ ] which can be seen as a generalized Fourior transformation of momentum space quark propagator in presence of local gluon field, another is a bilocal exponential "face factor" $\mathbf{a}[x-y ; A(x)]$. We now discuss them separately in detail.

For $\tilde{S}[x-y ; A(x)]$, note under gauge transformation $V(x), \nabla_{x}^{\mu}$ transform as $\nabla_{x}^{\mu} \rightarrow$ $V(x) \nabla_{x}^{\mu} V^{\dagger}(x)$ which leads $\tilde{F}^{\mu}\left(\nabla_{x}, \frac{\partial}{\partial k}\right) \rightarrow V(x) \tilde{F}^{\mu}\left(\nabla_{x}, \frac{\partial}{\partial k}\right) V^{\dagger}(x)$ and $\tilde{E}\left(\nabla_{x}, \frac{\partial}{\partial k}\right) \rightarrow$ $V(x) \tilde{E}\left(\nabla_{x}, \frac{\partial}{\partial k}\right) V^{\dagger}(x)$. (15) then tells us $\tilde{S}[z ; A(x)]$ take the transformation rule,

$$
\tilde{S}[z ; A(x)] \rightarrow V(x) \tilde{S}[z ; A(x)] V^{\dagger}(x)
$$

To calculate $\tilde{S}[x-y ; A(x)]$, we can first expand its integrand in terms of powers of commutators of $\nabla_{x}^{\mu}$, and denote $I_{n}\left(k, \frac{\partial}{\partial k} ; A\right)$ be n-th order of it, i.e.

$$
\left(\tilde{F}_{\partial k}+\not k+m\right)\left[\left(k+\tilde{F}_{\partial k}\right)^{2}+\tilde{E}_{\partial k}(x)-m^{2}\right]^{-1}=\sum_{n=0} I_{n}\left(k, \frac{\partial}{\partial k} ; A\right)
$$

with convention that $\frac{\partial}{\partial k_{\nu}}$ is always at r.h.s. of $k_{\nu}$. It is easy to find

$$
I_{0}\left(k, \frac{\partial}{\partial k} ; A\right)=\frac{\not k+m}{k^{2}-m^{2}}=\frac{1}{\not k-m},
$$

which is just free quark propagator in momentum space. Further with help of (11) and (13), we find $I_{1}\left(k, \frac{\partial}{\partial k} ; A\right)=0$ and 


$$
\begin{aligned}
& I_{2}\left(k, \frac{\partial}{\partial k} ; A\right) \\
& =\left\{\frac{i}{2} k_{\sigma} \gamma_{\rho} \gamma_{5} \epsilon^{\rho \sigma \mu \nu}-\frac{m}{4}\left[\gamma^{\mu}, \gamma^{\nu}\right]\right\} \frac{i g F_{\mu \nu}}{\left(k^{2}-m^{2}\right)^{2}}+\frac{\frac{i g}{2} \gamma^{\mu} F_{\mu \nu}}{k^{2}-m^{2}} \frac{\partial}{\partial k_{\nu}}-i g \frac{(\not k+m) F_{\mu \nu} k^{\mu}}{\left(k^{2}-m^{2}\right)^{2}} \frac{\partial}{\partial k_{\nu}} \\
& I_{3}\left(k, \frac{\partial}{\partial k} ; A\right) \\
& =\frac{g}{3} \gamma^{\mu}\left[\nabla_{x}^{\lambda}, F_{\mu \nu}\right]\left\{\frac{-2 g^{\lambda \nu}}{\left(k^{2}-m^{2}\right)^{2}}+\frac{8 k_{\nu} k_{\lambda}}{\left(k^{2}-m^{2}\right)^{3}}-\frac{2 k_{\nu}}{\left(k^{2}-m^{2}\right)^{2}} \frac{\partial}{\partial k^{\lambda}}-\frac{2 k_{\lambda}}{\left(k^{2}-m^{2}\right)^{2}} \frac{\partial}{\partial k^{\nu}}\right. \\
& \left.+\frac{1}{k^{2}-m^{2}} \frac{\partial^{2}}{\partial k^{\lambda} k^{\nu}}\right\}-\frac{g}{3}(\not k+m)\left[\nabla_{x}^{\lambda}, F_{\mu \nu}\right]\left\{\frac{1}{\left(k^{2}-m^{2}\right)^{3}}\left[-2 g^{\lambda \mu} k^{\nu}-4 g^{\lambda \nu} k^{\mu}-4 k^{\mu} k^{\lambda} \frac{\partial}{\partial k^{\nu}}\right]\right. \\
& \left.+\frac{1}{\left(k^{2}-m^{2}\right)^{2}}\left[g^{\lambda \mu} \frac{\partial}{\partial k^{\nu}}+2 k^{\mu} \frac{\partial^{2}}{\partial k^{\lambda} k^{\nu}}\right]\right\}+\frac{g}{2} \frac{(\not k+m) k_{\lambda}}{\left(k^{2}-m^{2}\right)^{3}}\left[\nabla_{x}^{\lambda},\left[\gamma^{\mu}, \gamma^{\nu}\right] F_{\mu \nu}\right] \\
& -\frac{g}{4} \frac{(\not k+m)}{\left(k^{2}-m^{2}\right)^{2}}\left[\nabla_{x}^{\lambda},\left[\gamma^{\mu}, \gamma^{\nu}\right] F_{\mu \nu}\right] \frac{\partial}{\partial k_{\lambda}} \\
& I_{4}\left(k, \frac{\partial}{\partial k} ; A\right) \\
& =-\frac{i g}{8}\left[\nabla_{x}^{\rho},\left[\nabla_{x}^{\lambda}, F_{\mu \nu}\right]\right]\left\{\frac{\gamma^{\mu}}{k^{2}-m^{2}} \frac{\partial^{3}}{\partial k^{\rho} \partial k^{\lambda} \partial k^{\nu}}+\frac{1}{\left(k^{2}-m^{2}\right)^{2}}\right. \\
& \left(-2 \gamma^{\mu}\left(g^{\lambda \nu} \frac{\partial}{\partial k^{\rho}}+g^{\nu \rho} \frac{\partial}{\partial k^{\lambda}}+g^{\rho \lambda} \frac{\partial}{\partial k^{\nu}}+k^{\rho} \frac{\partial^{2}}{\partial k^{\lambda} \partial k^{\nu}}+k^{\nu} \frac{\partial^{2}}{\partial k^{\rho} \partial k^{\lambda}}+k^{\lambda} \frac{\partial^{2}}{\partial k^{\rho} \partial k^{\nu}}\right)\right. \\
& \left.-(\not k+m)\left(2 k^{\mu} \frac{\partial^{3}}{\partial k^{\rho} \partial k^{\lambda} \partial k^{\nu}}+g^{\lambda \mu} \frac{\partial^{2}}{\partial k^{\rho} \partial k^{\nu}}+g^{\rho \mu} \frac{\partial^{2}}{\partial k^{\lambda} \partial k^{\nu}}+\left[\gamma^{\mu}, \gamma^{\nu}\right] \frac{\partial^{2}}{\partial k^{\rho} \partial k^{\lambda}}\right)\right) \\
& +\frac{1}{\left(k^{2}-m^{2}\right)^{3}}\left(2 ( \not k + m ) \left[g^{\rho \mu} g^{\lambda \nu}+g^{\rho \mu} k^{\nu} \frac{\partial}{\partial k^{\lambda}}+g^{\rho \mu} k^{\lambda} \frac{\partial}{\partial k^{\nu}}+2 k^{\mu} k^{\rho} \frac{\partial^{2}}{\partial k^{\lambda} \partial k^{\nu}}\right.\right. \\
& \left.+2 k^{\mu} k^{\lambda} \frac{\partial^{2}}{\partial k^{\rho} \partial k^{\nu}}+\left[\gamma^{\mu}, \gamma^{\nu}\right]\left(g^{\rho \lambda}+k^{\lambda} \frac{\partial}{\partial k^{\rho}}+k^{\rho} \frac{\partial}{\partial k^{\lambda}}\right)\right]+8 \gamma^{\mu}\left(g^{\rho \nu} k^{\lambda}+g^{\rho \lambda} k^{\nu}+g^{\nu \lambda} k^{\rho}\right. \\
& \left.+k^{\nu} k^{\lambda} \frac{\partial}{\partial k^{\rho}}+k^{\nu} k^{\rho} \frac{\partial}{\partial k^{\lambda}}+k^{\rho} k^{\lambda} \frac{\partial}{\partial k^{\nu}}\right)+2(\not k+m)\left(2 g^{\rho \nu} k^{\mu} \frac{\partial}{\partial k^{\lambda}}+2 g^{\rho \lambda} k^{\mu} \frac{\partial}{\partial k^{\nu}}+2 g^{\nu \lambda} k^{\mu} \frac{\partial}{\partial k^{\rho}}\right. \\
& \left.\left.+g^{\lambda \mu} g^{\rho \nu}+g^{\lambda \mu} k^{\nu} \frac{\partial}{\partial k^{\rho}}+g^{\lambda \mu} k^{\rho} \frac{\partial}{\partial k^{\nu}}\right)\right)+\frac{1}{\left(k^{2}-m^{2}\right)^{4}}\left(-48 \gamma^{\mu} k^{\rho} k^{\nu} k^{\lambda}\right. \\
& \left.\left.-8(\not k+m)\left(2 g^{\rho \nu} k^{\mu} k^{\lambda}+2 g^{\lambda \nu} k^{\mu} k^{\rho}+g^{\lambda \mu} k^{\nu} k^{\rho}+g^{\rho \mu} k^{\nu} k^{\lambda}+2 k^{\lambda} k^{\rho} k^{\mu} \frac{\partial}{\partial k^{\nu}}+\left[\gamma^{\mu}, \gamma^{\nu}\right] k^{\rho} k^{\lambda}\right)\right)\right\} \\
& -g^{2} F_{\lambda \rho} F_{\mu \nu}\left\{-\frac{1}{\left(k^{2}-m^{2}\right)^{2}}\left(\frac{1}{2} \gamma^{\lambda} g^{\rho \mu} \frac{\partial}{\partial k^{\nu}}+\frac{1}{2} \gamma^{\lambda} k^{\mu} \frac{\partial^{2}}{\partial k^{\rho} \partial k^{\nu}}+\frac{1}{8} \gamma^{\lambda}\left[\gamma^{\mu}, \gamma^{\nu}\right] \frac{\partial}{\partial k^{\rho}}\right.\right. \\
& \left.+\frac{1}{4}(\not k+m) g^{\lambda \mu} \frac{\partial^{2}}{\partial k^{\rho} \partial k^{\nu}}\right)+\frac{1}{\left(k^{2}-m^{2}\right)^{3}}\left(\gamma^{\lambda}\left(g^{\rho \mu} k^{\nu}+g^{\rho \nu} k^{\mu}+2 k^{\mu} k^{\rho} \frac{\partial}{\partial k^{\nu}}+\frac{1}{2} k^{\rho}\left[\gamma^{\mu}, \gamma^{\nu}\right]\right)\right. \\
& +\frac{1}{2} g^{\lambda \mu}(\not k+m)\left(g^{\rho \nu}+k^{\nu} \frac{\partial}{\partial k^{\rho}}+k^{\rho} \frac{\partial}{\partial k^{\nu}}\right)+k^{\lambda}(\not k+m)\left(g^{\rho \mu} \frac{\partial}{\partial k^{\nu}}+k^{\mu} \frac{\partial^{2}}{\partial k^{\rho} \partial k^{\nu}}\right) \\
& \left.+\frac{(\not k+m)}{4}\left(k^{\lambda}\left[\gamma^{\mu}, \gamma^{\nu}\right] \frac{\partial}{\partial k^{\rho}}+k^{\mu}\left[\gamma^{\lambda}, \gamma^{\rho}\right] \frac{\partial}{\partial k^{\nu}}+\frac{1}{4}\left[\gamma^{\lambda}, \gamma^{\rho}\right]\left[\gamma^{\mu}, \gamma^{\nu}\right]\right)\right) \\
& \left.-\frac{2}{\left(k^{2}-m^{2}\right)^{4}}(\not k+m)\left(g^{\lambda \mu} k^{\nu} k^{\rho}+g^{\rho \mu} k^{\nu} k^{\lambda}+g^{\rho \nu} k^{\mu} k^{\lambda}\right)\right\} \text {. }
\end{aligned}
$$


Correspondingly we can write

$$
\begin{aligned}
& \tilde{S}[z ; A(x)]=\sum_{n=0} \tilde{S}_{n}[z ; A(x)] \\
& \tilde{S}_{n}[z ; A(x)] \equiv \int \frac{d^{4} k}{(2 \pi)^{4}} I_{n}\left(k, \frac{\partial}{\partial k} ; A(x)\right) e^{-i k \cdot z}=\int \frac{d^{4} k}{(2 \pi)^{4}} I_{n}(k,-i z ; A(x)) e^{-i k \cdot z}
\end{aligned}
$$

After momentum integration, (23) and (24) will lead the expansion $\tilde{S}[z ; A(x)]=$ $\sum_{n} C_{n}(z) O_{n}[A(x)]$ mentioned previously in (3). The leading term $\tilde{S}_{0}[z ; A(x)]=C_{0}(z)$ with $O_{0}[A(x)]=1$ is just the exact propagator in the trivial case of vanishing gluon field,

$$
\tilde{S}_{0}[z ; A(x)]=\int \frac{d^{4} k}{(2 \pi)^{4}} \frac{e^{-i k \cdot z}}{\not k-m} .
$$

Now, we come to discuss the exponential "face factor", it satisfy constraints

$$
\begin{aligned}
& \mathbf{a}[0 ; A(x)]=1 \quad \mathbf{a}[x-y ; 0]=1 \\
& (x-y) \cdot \nabla_{x} \mathbf{a}[x-y ; A(x)]=\left.\left[z \cdot \nabla_{x}+z \cdot \partial_{z}\right] e^{-z \cdot \nabla_{x}} 1\right|_{z=x-y}=0 .
\end{aligned}
$$

In the literature, these constraints usually lead to path ordered non-integratable face factor $\mathbf{P} e^{i g \int_{y}^{x} d z^{\mu} A_{\mu}(z)}[6]$ which depend on path. Our result instead only rely on end points $x, y$ and is independent of the path. Except the formal definition of $\mathbf{a}[x-y ; A(x)]$, the explicit expression of $\mathbf{a}[x-y ; A(x)]$ can be got with help of Baker-Housdoff formula

$$
\mathbf{a}[x-y ; A(x)]=\left.\left[e^{-z \cdot \nabla_{x}} e^{z \cdot \partial_{x}} 1\right]_{z=x-y} \equiv e^{C(x, z)}\right|_{z=x-y},
$$

where $C(x, z)$ is defined as

$$
\begin{aligned}
e^{C(x, z)}= & e^{-z \cdot \nabla_{x}} e^{z \cdot \partial_{x}}=\exp \left[-z \cdot \nabla_{x}+z \cdot \partial_{x}+\frac{1}{2}\left[-z \cdot \nabla_{x}, z \cdot \partial_{x}\right]+\frac{1}{12}\left[-z \cdot \nabla_{x},\left[-z \cdot \nabla_{x}, z \cdot \partial_{x}\right]\right]\right. \\
& \left.-\frac{1}{12}\left[z \cdot \partial_{x},\left[z \cdot \partial_{x},-z \cdot \nabla_{x}\right]\right]+\cdots\right] \\
= & \exp \left[i g z \cdot A(x)+\frac{1}{2}\left[i g z \cdot A(x), z \cdot \partial_{x}\right]+\frac{1}{12}\left[i g z \cdot \nabla_{x},\left[i z \cdot A(x), z \cdot \partial_{x}\right]\right]\right. \\
& \left.-\frac{1}{12}\left[z \cdot \partial_{x},\left[z \cdot \partial_{x}, i g z \cdot A(x)\right]\right]+\cdots\right]
\end{aligned}
$$

Note $C(x, z)$ donot include pure operator $\partial_{x}$ again, all $\partial_{x}$ in $C(x, z)$ are already acting on gluon filed $A_{\mu}(x)$ and gluon field dependence in $C(x, z)$ is local at space-time point $x$.

The gauge transformation covariance of $\mathbf{a}[x-y ; A(x)]$ can be proved as follows: since under gauge transformation $V(x), \nabla_{x}^{\mu}$ transform as $\nabla_{x}^{\mu} \rightarrow V(x) \nabla_{x}^{\mu} V^{\dagger}(x), \mathbf{a}[x-y ; A(x)]$ then transform as 


$$
\begin{aligned}
\mathbf{a}[x-y ; A(x)] & \rightarrow\left[e^{-V(x) z \cdot \nabla_{x} V^{\dagger}(x)} 1\right]_{z=x-y}=V(x)\left[e^{-z \cdot \nabla_{x}} V^{\dagger}(x)\right]_{z=x-y} \\
& =V(x)\left[e^{-z \cdot \nabla_{x}} e^{z \cdot \partial_{x}} e^{-z \cdot \partial_{x}} V^{\dagger}(x)\right]_{z=x-y}=V(x) e^{C(x, z)}\left[e^{-z \cdot \partial_{x}} V^{\dagger}(x)\right]_{z=x-y} \\
& =\left[V(x) e^{C(x, z)} V^{\dagger}(x-z)\right]_{z=x-y}=V(x) \mathbf{a}(x, y ; A) V^{\dagger}(y),
\end{aligned}
$$

where we have used property

$$
e^{-z \cdot \partial_{x}} V^{\dagger}(x)=\left[1+\sum_{n=1}^{\infty} \frac{(-1)^{n}}{n !} z_{\mu_{1}} \cdots z_{\mu_{n}} \partial_{x, \mu_{1}} \cdots \partial_{x, \mu_{n}}\right] V^{\dagger}(x)=V^{\dagger}(x-z)
$$

Combine (17) and (29) together, we find our result quark propagator (14) is gauge transformation covariant

$$
S(x, y ; A) \rightarrow V(x) S(x, y ; A) V^{\dagger}(y)
$$

Similarly since the Lorentz covariance for $\tilde{S}$ and a is explicit, our result propagator is explicit Lorentz covariant.

In conclusion, we have factorized the quark propagator $S(x, y ; A)$ by a generalized Fourior transformation of momentum space quark propagator $\tilde{S}[x-y ; A(x)]$ in presence of gluon field and a path independent "face factor" $\mathbf{a}[x-y ; A(x)]$. The two parts are all only depend on gluon field at local space-time point $x$. The formalism is gauge and Lorentz covariant, it coincide with the exact propagator in the trivial case of vanishing gluon field.

\section{ACKNOWLEDGMENTS}

This work was supported by National Science Foundation of China No.90103008 and fundamental research grant of Tsinghua University. 


\section{REFERENCES}

[1] L.S.Brown, W.I.Weisberger, Phys. Rev. D20, 3239(1979).

[2] E.Eichten, F.Feinberg, Phys. Rev. D23, 2724(1981).

[3] W.Lucha, F.F.Schoberl, D.Gromes, Phys. Rep. 200, No4, 127(1991).

[4] D. Gromes, Eur. Phys. J, C20, 523 (2001).

[5] W. Miller, Jr, Symmetry Groups and Their Applications, Academic Press 1972.

[6] R. D. Ball, Phys. Rep. 182 (1989)1. 